\title{
Analysing MANET Ability to work with WSN in IoT Environment using IPv6 (Information Harvester Scenario)
}

\author{
Mahmood Ibrahim Alsaydia \\ MSc. University of Salford \\ Greater Manchester
}

\begin{abstract}
Next Generation Networks (NGN) have brought Mobile Adhoc Networks (MANETs) back to the forefront. Since, that everything is managed to be linked to the internet (IoT), heterogeneous networks have been presented as the key factor in NGN for enabling the most benefits that can be realized through collaboration between a diversity of devices working with different standards but all connected to the internet. All that makes the cogitation focusing on infrastructure less networks. Additionally, with the availability of IP addressing in IPv4 running out, IPv6 has emerged to be considered the fundamental protocol to be used in NGN. Since Wireless Sensor Networks (WSN) are considered to be a part of Ad-hoc networks, though working on a different standard, an opportunistic integration between MANET and WSN has been achieved as one of the aspects of IoT. Where, via configuring nodes named Multi Purpose Devices (MPDs) to work on both MANET and WSN standards, connections have been established, in the design that had been called information harvester $(\mathrm{IH})$, between sensors transmitting data to one of MANET nodes (sink) and vice versa. Moreover, the number of packets delivered to the sink node increased due to mobility of these MPDs.
\end{abstract}

\section{Keywords}

Mobile Ad-hoc NETwork (MANET), Wireless Abstract Link MAC (WALM), Multi-Purpose Device (MPD), Wireless Sensor Network (WSN).

\section{INTRODUCTION}

Currently, more than five billion of PDAs, Mobiles, computer and many other devices are connected to the internet, primarily wirelessly, and through a diverse and combined set of networks, like $3 \mathrm{G}$ and $4 \mathrm{G}$ cellular networks, as well as WiFi with its different standards, for example, Mobile Ad-hoc Networks (MANETs).

Likewise, the vision of the next generation networks considers interoperability between the aforementioned networks and the unmentioned, too. Presenting what has become known as heterogeneous networks [1]. This gives the opportunity to connect and control things, in addition to connect people, such as ordinarily daily used devices and machines, from cars to a variety of home and work equipment, and so on. In addition, it potentially allows sensing the surrounding environment in order to give better proactive information about many factors, like gas emissions, fire alarms, and healthcare information. These factors and more are pushing us toward living in smart cities and producing what is becoming known as the Internet of Thing (IoT) [2]. Moreover, this has strongly brought back Ad-hoc network technology, which does not rely on fixed base stations to initiate communication among mobile nodes, to the [3].
With such a novel network, the ability to come up with a solution for many problems in the performance of individual networks can be realised, such as information harvesting challenges in Wireless Sensor Networks (WSN), which considered as a branch of Ad-hoc networks. On the other hand, due to IPv4 address exhaustion, meaning running out of the availability of Internet Protocol version four (IPv4) address allocations because of the skyrocketing number of mobile devices connected to the internet. IPv6, which has been presented by the Internet Engineering Task Force (IETF) early in 1994, will emerge to be used instead of IPv4 by 2020, that is the expected date the world will ultimately be moved to Next Generation Networks (NGN). IPv6 uses a 128-bit addressing size in comparison to the 32-bit addressing size in IPv4, who is on its way to being considered as a legacy protocol, IPv6 can be allocated to $3.4 \times 10^{38}$ devices without any need to use Network Address Translation (NAT), against 4.4 billion devices for IPv4 [4]. Hence, any talk about NGN directs the mind to IPv6, which will be used in this paper. The rest of this paper is organized as follows. Section II briefly introduces the two IEEE 802 standards. Section III.

Discusses some of the related literature about MANET-WSN network. While Section IV explain the design and the implementation. Section V reports extensive experimental results about the IH scenario. And finally, in section VI, conclusive marks, and directions of future work end the paper.

\section{STANDARDS}

MANET usually works with IEEE 802.11 standard, which operates on the $2.4 \mathrm{GHz}$ and $5 \mathrm{GHz}$ Industrial, Science and Medical (ISM) frequencies, and comes in many different variations, such as IEEE $802.11 \mathrm{a} / \mathrm{b} / \mathrm{g} / \mathrm{n}$ [5]. While WSN works with IEEE 802.15.4 Standard. The best commonly known example of it is ZigBee, which is a low tier, Ad-hoc, terrestrial wireless standard that is in some way similar to Bluetooth. ZigBee has some features in addition to those of 802.15.4. It operates in the $868 \mathrm{MHz}, 915 \mathrm{MHz}$ and $2.4 \mathrm{GHz}$ ISM bands [6].

Although they are both considered as Ad-hoc networks, the physical and MAC layers of these two standards are not compatible [7]. IEEE 802.11 standard defines both the physical (PHY) and Medium Access Control (MAC) layer protocols for WLANs. The 14 channels available in the 2.4 $\mathrm{GHz}$ band have been specified for this standard, with $22 \mathrm{MHz}$ bandwidth for each channel, separated by $5 \mathrm{MHz}$. These channels' mapping can be seen in Figure 1 [8], which compares these two standards' channels. WLAN output powers are typically around $20 \mathrm{dBm}$ and operate within a $100 \mathrm{~m}$ range for the legacy standards and a wider range in the new standards. The contention channel scheme in MAC layer used by all IEEE 802.11 protocols (IEEE $802.11 \mathrm{~b} / \mathrm{g} / \mathrm{n}$ ) is Carrier Sense Multiple Access with Collision Avoidance (CSMA/CA). The modulation technique is Binary Phase Shift 
Key (BPSK) in additional to Orthogonal Frequency Division Multiplexing (OFDM) in new generations like $802.11 \mathrm{~g}$, which was absent in the initial IEEE $802.11 \mathrm{~b}$ standard. This allows an increase in the maximum physical layer bit rate of up to 54 Mbps from the $11 \mathrm{Mbps}$ used by the earlier $802.11 \mathrm{~b}$ standard utilizing DSSS modulation [8].

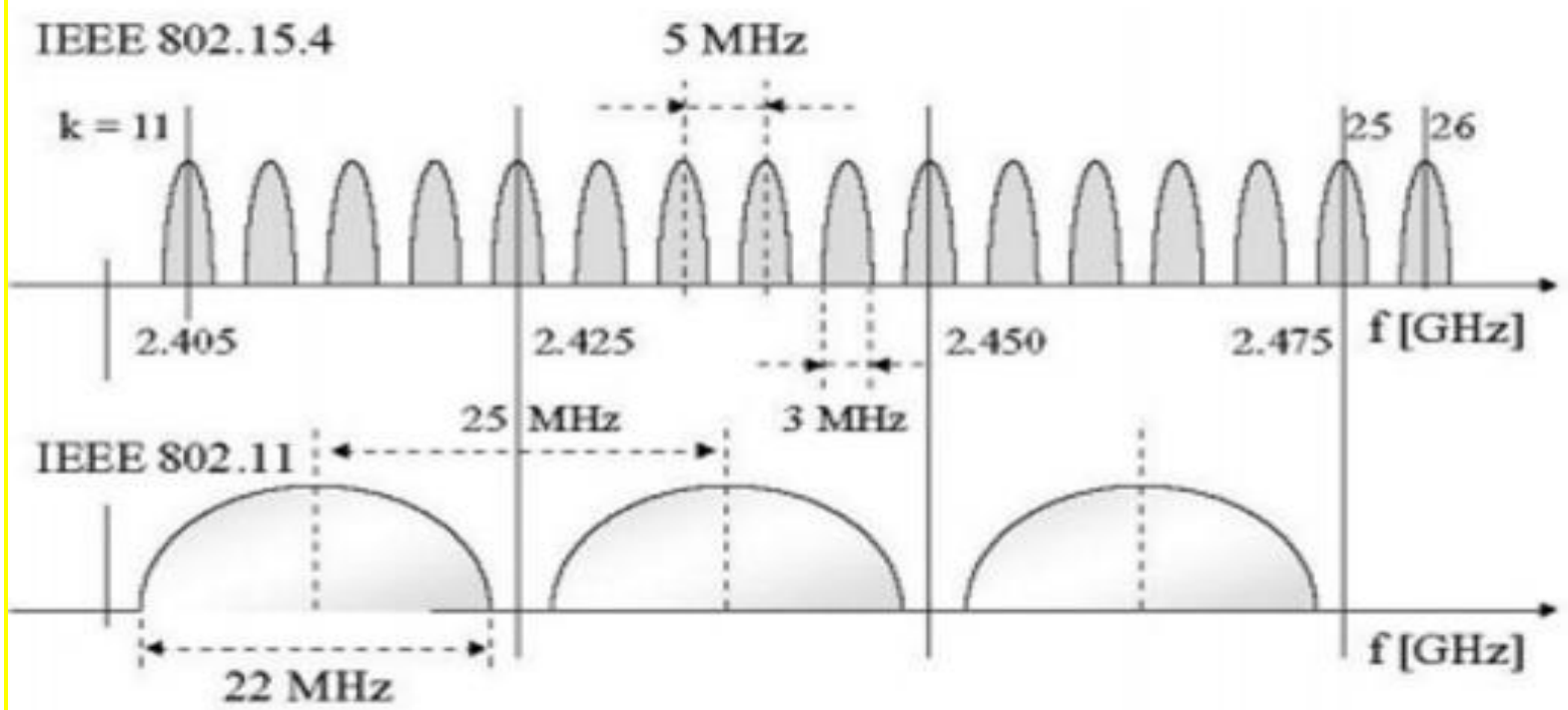

Fig 1: Frequency channels of IEEE 802.15.4 compared with IEEE 802.11

On the other hand, IEEE 802.15.4 standard defines both PHY layer and MAC sublayer specifications for low-rate wireless personal area networks (LR-WPANs), where usually simple, low power consumption devices work in about a 10-meter area. The PHY is based on Direct Sequence Spread Spectrum (DSSS) techniques, and the data rate is about $250 \mathrm{kbps}$ at 868 $\mathrm{MHz}$ using BPSK modulation techniques, and lately, OFDM for a higher bitrate. The channels used for this standard can be seen in Figure 1. Also, the CSMA/CA mechanism is used to access these channels [6][8].

It is significant to know that IEEE 802.15.4 defines 127 bytes as the maximum length of the MAC header, as Table 1 illustrates, while 802.11 usually works with IP and can carry the even long header of IPv6 without needing an extra special mechanism, as the former needs, due to compressions and the use of the new IETF standard that is known as 6LoWPAN. Where 6LoWPAN allows IEEE 802.14.5 header to work with IPv6 via a special technique. From the previous figure, it can clearly be seen that interference usually occurs whenever both standards work in the same area. Yet in contrast, potential cooperation can be exploited through their commonalities, under special configuration, as what we have done in this paper.

\section{TABLE 1. IEEE 802.15.4 header}

\begin{tabular}{|c|c|c|}
\hline 5 Octets & 1 Octet & Maximum 127 Octets \\
\hline $\begin{array}{c}\text { Sync } \\
\text { Header }\end{array}$ & PHY & PHY Payload (PSDU) \\
\hline
\end{tabular}

\section{LITERATURE RIVIEW}

In a smart urban scenario where sensors are supposed to be deployed widely and over almost everything, supplying data collected from intelligent devices. In such a perspective, a collaboration and integration between the different networks' technologies and different standards are essential. Addressing this inevitability in NGN, it can be seen that MANET and WSN, despite the incompatibility between their PHY and MAC layers, as what has been previously mentioned, can collaborate. Thereon, research [9], came up with the suggestion that the two IEEE 802 standards need to be modified and matched to enable them to work directly together under all conditions and in both directions. Furthermore, their study presented a suggested protocol called MANET coordination protocol, which is based on a collaborative mechanism when there is an urgent packet that needs to be delivered. The researchers tested their proposed work through both real deployment and simulation. Within the simulation and by using QualNet, several sensor nodes had been distributed in two specific smart city scenarios. One was a street $1 \mathrm{~km}$ long and $10 \mathrm{~m}$ wide monitored by 50

Sensors separated by $20 \mathrm{~m}$ apart, and the other was an area $1 \times 1 \mathrm{~km}$ monitored by 200 sensors. With each scenario, a different number of MANET nodes had been distributed, clustered and configured to run over IEEE $802.11 \mathrm{~b}$ standard. A modification to the PHY layer in IEEE 802.15.4 had been done, by running MICAz energy model, and then researchers measured power consumption, latency, and delivered packet ratios when both normal and urgent packets were sent, in order to examine how much power consumptions could be reduced when only the urgent packets would be sent.

Another collaboration assumption has been presented in a disaster environment by (Bai, Du, Ma, Shen, Zhou \& Chen, 2010), where an Emergency Communication System formed by heterogeneous wireless networking, as shown in Figures 2 and $3[10]$. This study assumed that the sink node in WSN system deployed in a disaster area could be connected to one of the MANET nodes when the latter had already been deployed in such environment due to the lack of network infrastructure. The sink node, in this case, would be

connected to the MANET nodes through an Access Point (AP), and must have the ability to work with both standards. 
MANET, in turn, would be connected to either a cellular or a satellite gateway in order to transmit crucial data to an emergency information centre.

From these collaboration perspectives, a different model, called Information Harvester (IH) will be designed and implemented in a smart urban scenario in our project. In it, as what will be seen later, under a special technique, IEEE 802.11 standard can receive packets from IEEE 802.15.4 standard, and vice versa.

The last literature [11], investigate in the reliable communication for MANET-WSN scenario. Although researchers assumed a scenario for transmitting urgent data from sensors to MANET, but their investigating was only from WSN perspective using Link Quality Indicator (LQI) and Received Signal Strength Indicator (RSSI), testing the reliability of an assumed heterogeneous network (MANETWSN) statically and through mobility. Nevertheless, there was no MANET deployment in the scenario. Different to what have been done in [11], we deployed MANET node and involved some indicators to measure the reliability of the connection. Moreover, how it can be enhanced and accelerated.

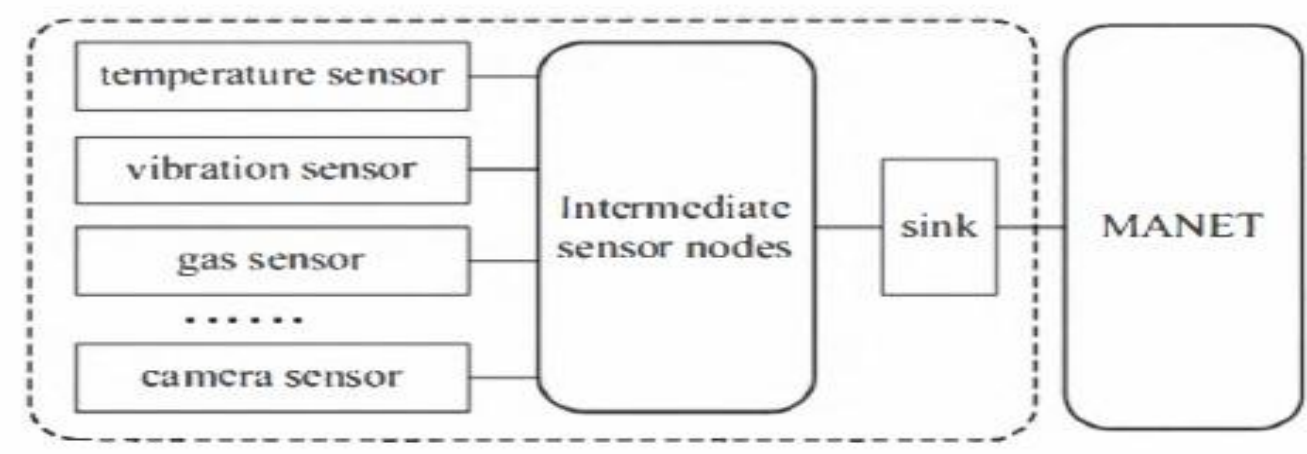

Fig 2: WSN sink node connected to MANET

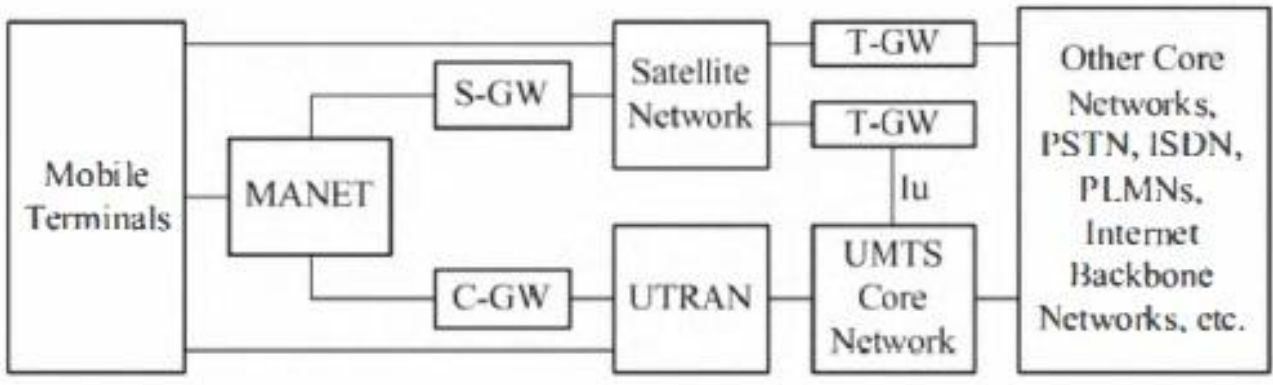

Fig 3: MANET connected to Gateways

\section{SIMULATION OF CONVERGING MANET AND WSN IN A SMART URBAN (INFORMATION HARVESTER DESIGN)}

The two perspective that we successfully implemented in our work was establishing connection and data transmission between WSN nodes and MANET node by using two techniques which they were firstly, using MPDs, and secondly, providing WALM, as can figure 4 clarify. The real implementation was simulating an urban environment taking into consideration the realistic distribution of the sensors over a specific area as what will be seen later. Although we have not used 6LoWPAN, but we succeed to establish the connection over IPv6 by using the fragmentation techniques. As it is known, in any WSN system that works on IEEE 802.15.4 standard, devices can work in one of two functions, which are Full Function Device (FFD) or Reduced Function Device (RFD). The former has three modes, Coordinator, Device, and Personal Area Network Coordinator (PAN Coordinator). The destination node (sink node), where the collected information used to be transferred to, must be configured as a PAN coordinator. The other nodes (those that sense the environment) are usually configured to work in RFD. Where FFD can transmit, and receive from all other modes, but an RFD node cannot transmit and receive from another RFD node [12].

As the maximum length of the header for this standard is 127 bytes, then the item packet size of the CBR connection must be chosen carefully to prevent the whole transmitted packet size from exceeding 127 bytes, leading to it being dropped. Therefore, it will be chosen to be 70 bytes.

When it comes to the convergence of the two previously mentioned standards, a node working on IEEE 802.11 can send or receive packets to or from any node working on IEEE 802.15.4 indirectly, but through a preconfigured node that can work on both standards, and it will be called a Multiple Purpose Device (MPD). Hence, any IEEE 802.11 standard node that receives, as a final destination, packets from plenty of sensor nodes can be considered a sink node [10], as in military applications (battlefield networks). In such a scenario, MPDs must be able to sense and listen to the radio channels that both standards use. Therefore, in the simulation, two channels need to be configured. One of them will be set to work on $2.4 \mathrm{GHz}$; the other will be set to $2.41 \mathrm{GHz}$, as in Figure 5. That means that MPDs will work, as they have double antennas, or antennas that can switch to work over the two standards, and this will prevent collisions. 


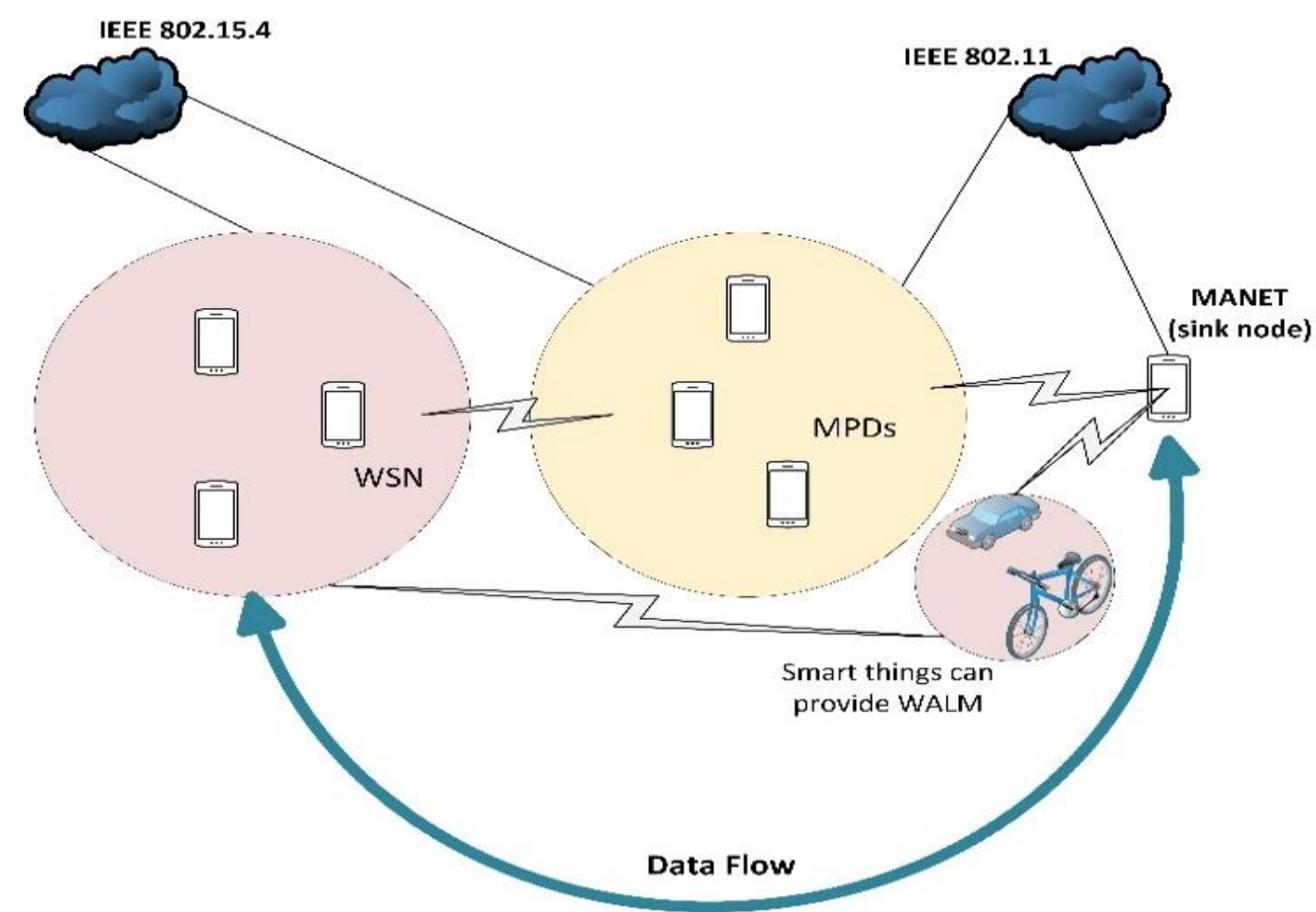

Fig 4: MANET-WSN NETWORK by using MPDs and WALM
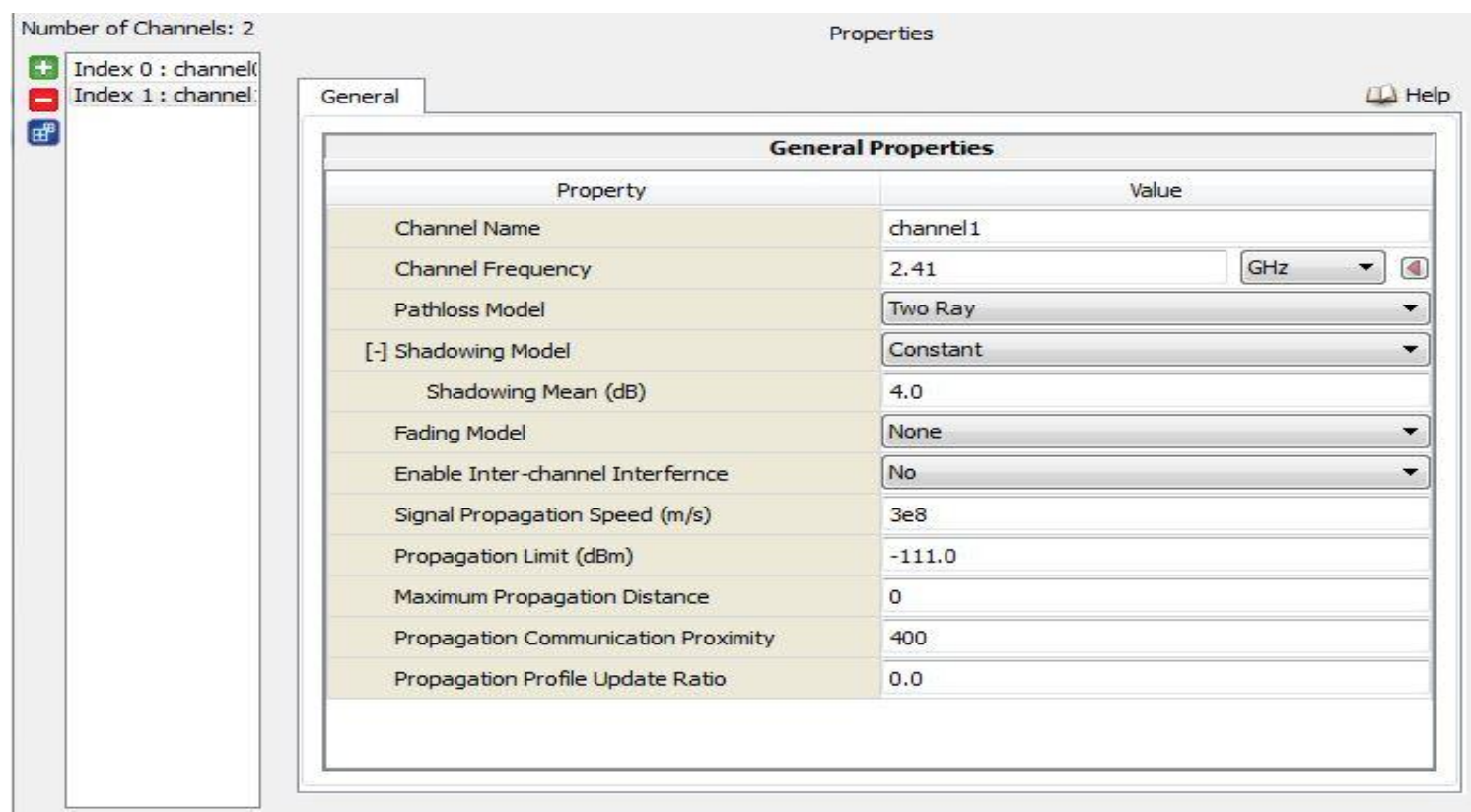

\section{Fig 5: Channels configuration}

Likewise, MPDs must be configured to work as PAN coordinators on IEEE 802.15.4 side, as Figure 6 shows, while from the 802.11 side, the normal configuration will be set, as in the aforementioned configuration. While, the whole distributed sensors' MAC layer will be configured to work as RFD. In this case, these MPDs will receive packets that need to be sent from WSN sensor nodes to IEEE 802.11 node (sink node), and then will deliver them to the latter when they become in both standards devices' coverage area, and vice versa. That is, if MPDs move between the former and the latter, and they will act similarly if they were static between WSN and IEEE 802.11 nodes in which they can connect.
As can be seen in Figure 7, a design has been created to simulate such an integration, as would be found in an urban scenario, where 38 sensor nodes have been distributed over buildings and in streets, and then 10 MANET nodes have been distributed randomly and configured as MPDs. In additional, their mobility management has been modified to make them move as if in an urban environment. Another static MANET node will act as a sink node and receive information from some of the distributed sensors. 


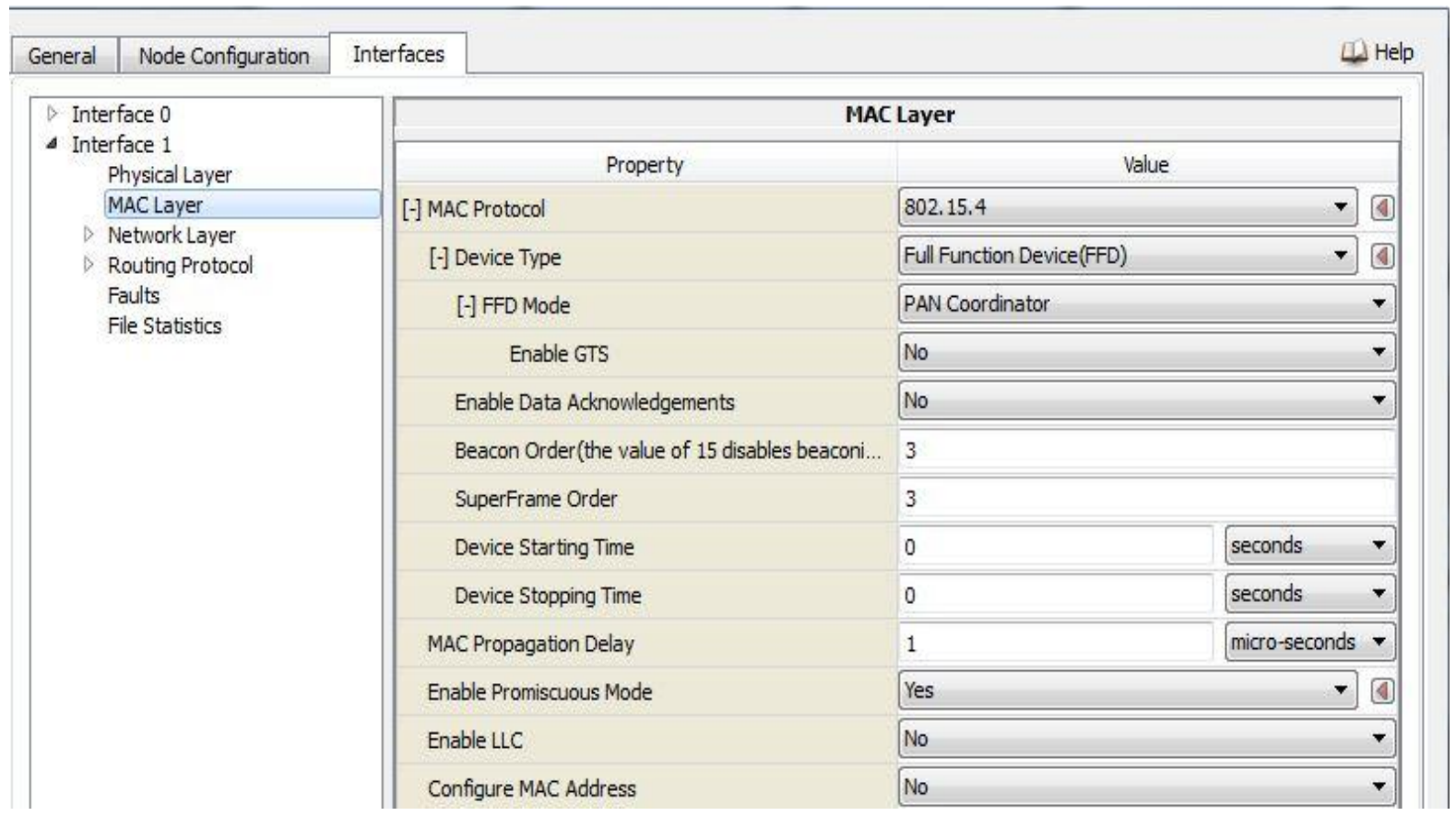

Fig 6: MAC configuration for MPDS on 802.15.4

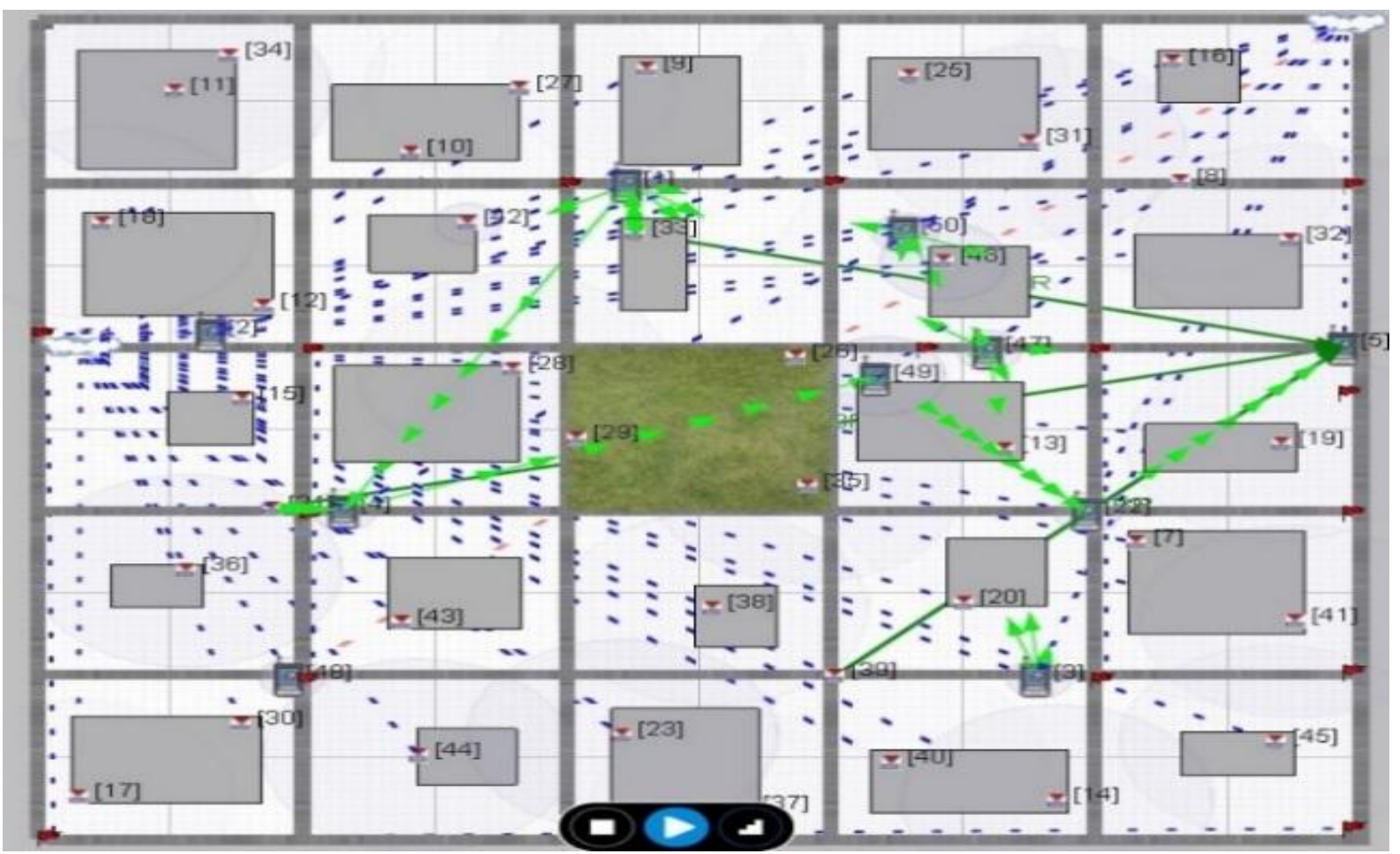

Fig 7: MANET and WSN in urban environment 


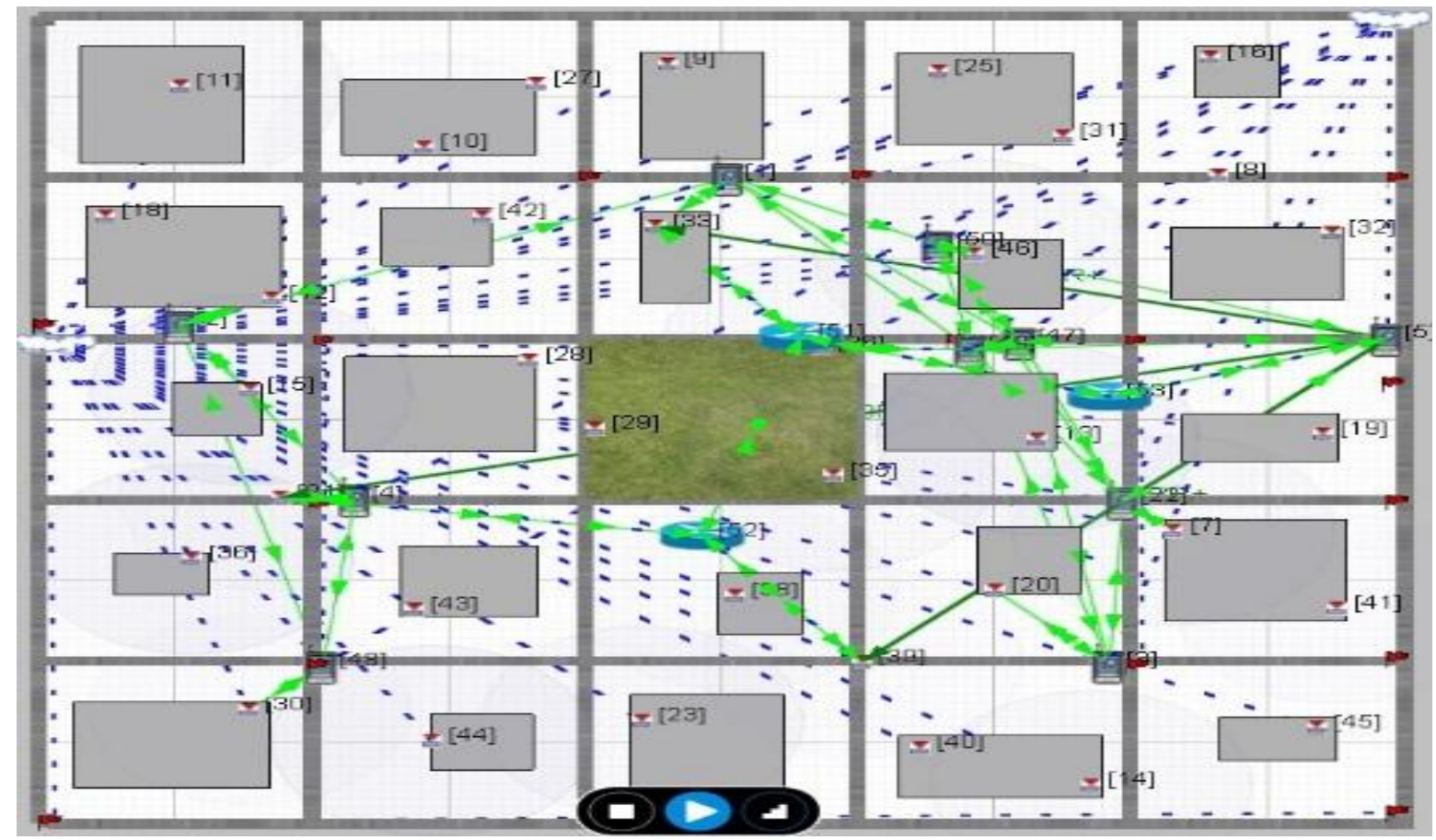

Fig 8: MANET and WSN and Wireless Abstract link MAC provider in urban environment

This kind of design can be considered as another aspect of the smart city environment. After implementing, running, collecting and analysing the results, an assumption of providing Wireless Abstract Link Mac (WALM), by using devices that have been configured to provide it, had been implemented, to improve the reliability of the network. In this novel design, and in the smart urban concept, let us imagine that a car, or a person or any smart thing, can act as this assumed node, and it moves between the destination mobile node (sink node) and the transmitting nodes. And then during its appearance between the transmitting nodes and destination nodes it can accelerate the transmission to increase the reliability of the overall transmitting, as in figure 8 and the result show. Also, it is necessary to mention that IEEE 802.15.4 standard cannot work with IPv6 unless special configurations and techniques are used. Where, as had been mentioned previously, 6loWPAN is the standard that can provide one of those techniques.
Since this standard is not supported by QualNet, then a very precise and careful configuration was done to avoid the disability of WSN and MANET to work together over IPv6. Where the network layer of the cloud that connect the sensors has been configured as Figure 9 shows, while the one for the cloud that the MANET nodes connected to, and similarly, the sensor nodes, has been configured as seen in Figure 10.

In both of these implementations, DYMO routing protocol was chosen to be applied because of the satisfactory results through most of the MANET routing protocols studies. Besides, it's ability to deal with multiple gateways.

Finally, we repeated the last scenario, but another CBRs have been applied to transmit data back to the three sensors from the sink node (node 5), and the packets size were set to 512 bytes, in order to test whether or not the WALM provided by the assumed devices could make the sensors' node receive directly from the sink node, as well as to test the ability to increase the size of the transmitted packet. 


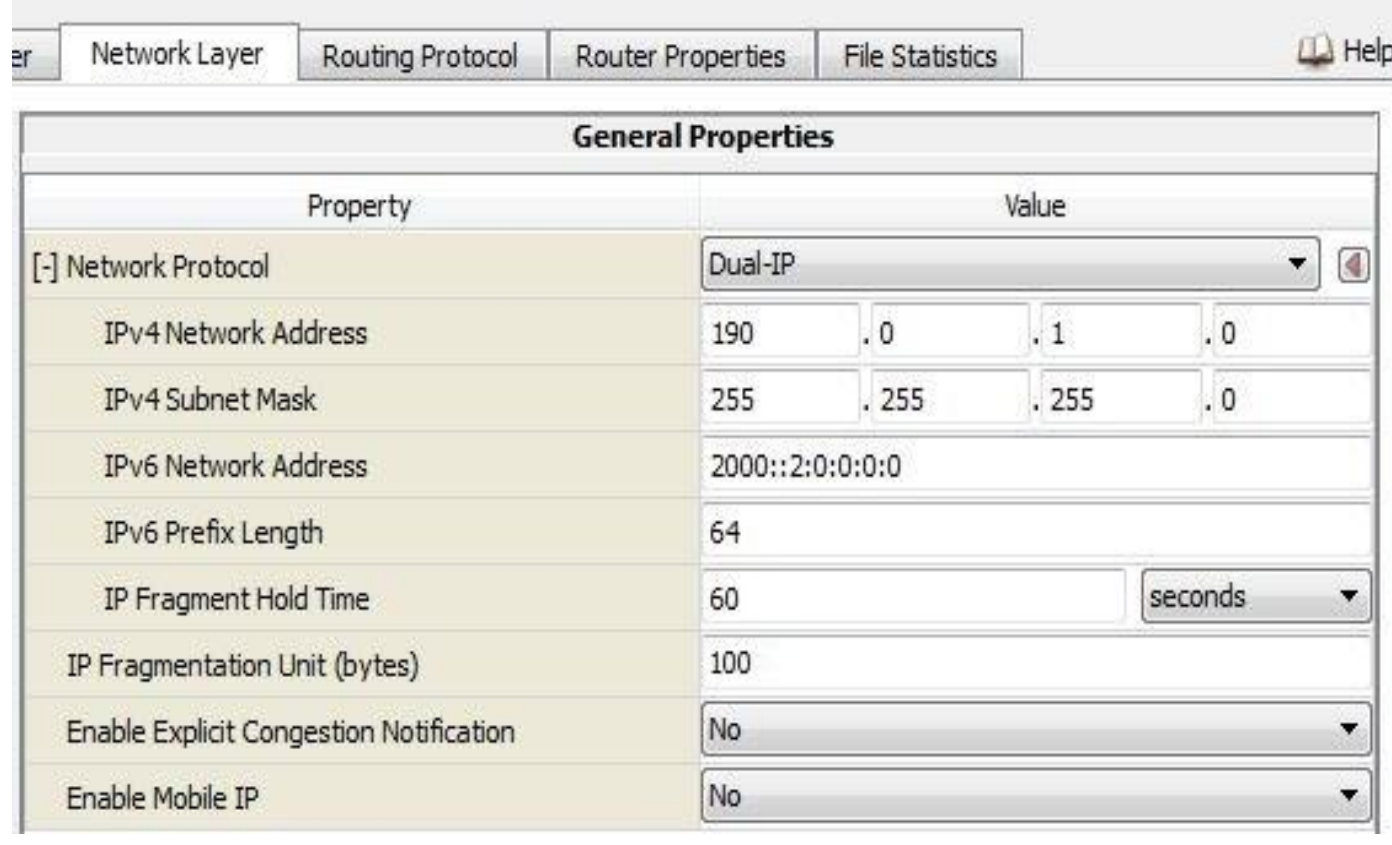

Fig 9: Network layer configuration for IEEE 802.15.4 CLOUD in the Information Harvester Design

\begin{tabular}{|c|c|c|c|c|c|c|}
\hline General & Node Configuration & \multicolumn{2}{|l|}{ Interfaces } & & \multicolumn{2}{|c|}{4 Help } \\
\hline \multirow{18}{*}{\multicolumn{2}{|c|}{$\begin{array}{l}\text { Mobility and Placement } \\
\text { Network Layer } \\
\text { Routing Protocol } \\
\text { Router Properties } \\
\text { Transport Layer } \\
\text { MPLS } \\
\text { Application Layer } \\
\text { User Behavior Model } \\
\text { Battery Model } \\
\text { External Interface Properties } \\
\text { Faults } \\
\text { File Statistics } \\
\text { Statistics Database } \\
\text { Packet Tracing } \\
\end{array}$}} & \multicolumn{5}{|c|}{ Network Layer } \\
\hline & & \multicolumn{2}{|r|}{ Property } & \multicolumn{3}{|c|}{ Value } \\
\hline & & \multicolumn{2}{|c|}{$[-]$ Network Protocol } & \multicolumn{2}{|l|}{ IPv6 } & (4) \\
\hline & & & Enable IPv6 Autoconfiguration & \multicolumn{2}{|l|}{ No } & $\rightarrow$ \\
\hline & & & IP Fragment Hold Time & 60 & \multirow[t]{2}{*}{ seconds } & $\rightarrow$ \\
\hline & & & Fragmentation Unit (bytes) & 100 & & (4) \\
\hline & & & able Explicit Congestion Notification & \multicolumn{2}{|l|}{ No } & $\rightarrow$ \\
\hline & & {$[-] \mathrm{En}$} & able ICMP & \multicolumn{2}{|l|}{ Yes } & $\checkmark$ \\
\hline & & & Is ICMP Router? & \multicolumn{2}{|l|}{ No } & - \\
\hline & & & Enable Redirect & \multicolumn{2}{|l|}{ Yes } & 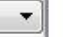 \\
\hline & & & Redirect Retry Time & 1 & seconds & 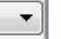 \\
\hline & & & Allow Override of Non-Static Routes & \multicolumn{2}{|l|}{ Yes } & - \\
\hline & & & Router Advertisement Life Time & 1800 & seconds & - \\
\hline & & & Minimum Router Advertisement Interval & 450 & seconds & \\
\hline & & & Maximum Router Advertisement Interval & 600 & seconds & - \\
\hline & & & Maximum Number of Solicitation & \multicolumn{3}{|l|}{3} \\
\hline & & & Enable ICMP Error Messages & \multicolumn{2}{|l|}{ No } & 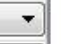 \\
\hline & & & able Mobile IP & \multicolumn{3}{|l|}{ No } \\
\hline
\end{tabular}

Fig 10: Network layer configuration for IEEE 802.11 CLOUD and NODES in the Information Harvester Design

\section{ANALYZING THE SIMULATIONS AND OUTCOMES}

After implementing all the designs in QualNet and running each of them more than ten times, data and outcomes have been collected to be discussed, and analysed in the next part of this paper. Additionally, IPv6 fragmentation had been activated and was set to lower than 127 bytes. Decreasing the data packet size to 70 bytes gave space to the routing protocols information to be added within this limited size header, which is 127 bytes. Furthermore, the cloud that connects nodes, which works on IEEE 802.15.4 standard, was configured to allow tunneling IPv6 packets over IPv4.

As in figures $(7,8)$ which show the scenarios through their running. All scenarios succeeded in presenting collaborative work that could be utilized in IoT, but the results were disparate, as shown in Figure 11. The unicast messages received from the three sensors to node 5 were very low when the MPDs were set to be statics, where only about 10 messages out of 100 over each sensor were delivered, while when the MPDs were set to be mobile nodes. The total number of messages received increased from 31 to 66 out of 300 transmitted messages, and there was a failure to transmit any packet at all from one of the three sensors. 


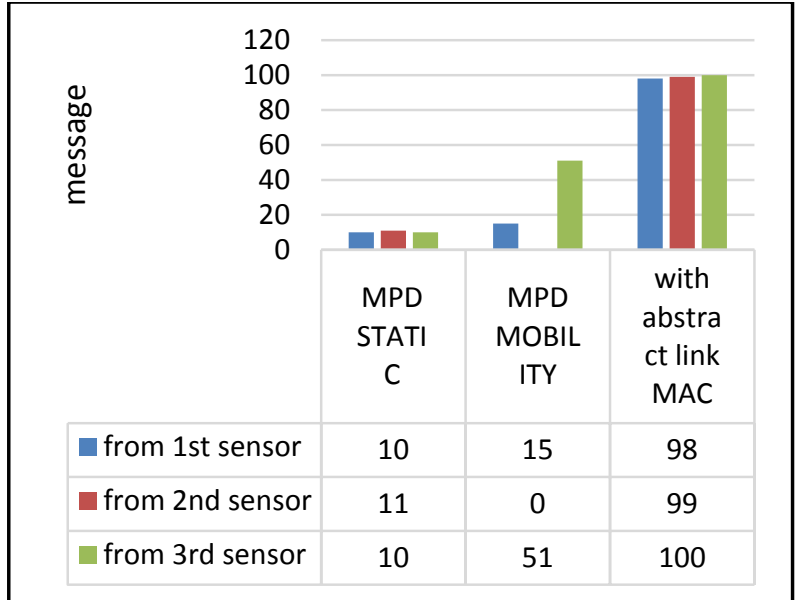

Fig 11: Unicast Messages Received from each Sensor in each Scenario

When three nodes provided the same assumption of WALM, the total unicast messages received rose strongly to 297 messages, whilst the AE2ED decreased for the first sensor when the MPDs were mobile, to about 2.04 seconds, while it was 6.315 seconds when the MPDs were static. Moreover, a slight decrement of about 0.1 second can be seen when it came to the third sensor, as Figure 12 shows, which again was formatted logarithmically to scale 10 , to clarify the differences and the small numbers.

In contrast, a remarkable decline had been noticed in AE2ED when the assumption nodes with WALMs were deployed. That led to a significant gain in the unicast received throughput; it jumped up to around $565 \mathrm{bits} / \mathrm{sec}$ when the assumed WALM had been provided by the three deployed nodes, while it was around $20 \mathrm{bits} / \mathrm{sec}$ when all MPDs were static, as Figure 13 clarifies. One of this explicit enhanced in the performance reasons was because of these three nodes were set to be static, and the actual enhancement might be a bit less than what has been found if these nodes were mobile.

The low throughputs found with this design were caused by the small packet size that has been configured to enable the integration between WSN and MANET. Where, as what has been discussed previously, the limitation came from the limit of 127 bytes for the maximum header length for IEEE802.15.4 standard, while the IPv6 mechanism is obliged to transmit 1280 bytes in its header as a minimum, even if it is not fully occupied [4], if fragmentation is disabled. Therefore, fragmentation has been enabled in this design, and its threshold was set to 100 bytes.

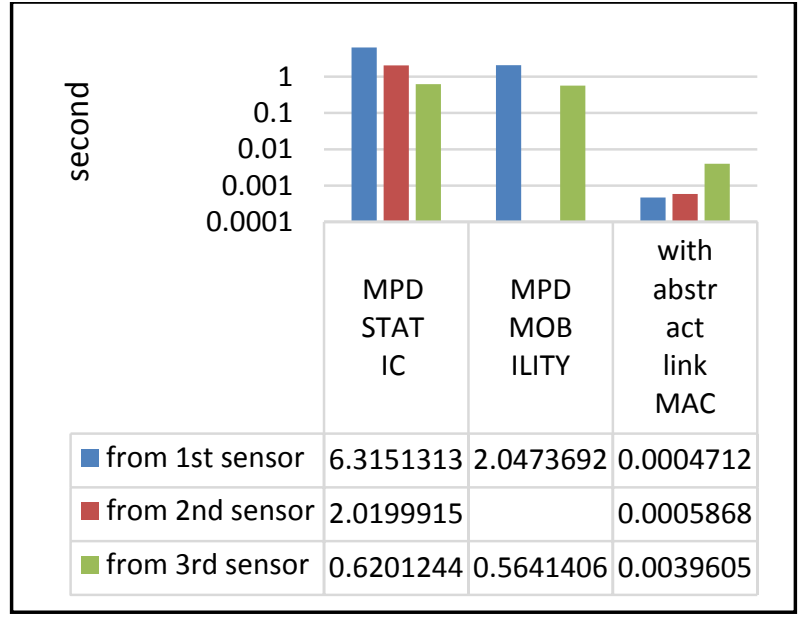

Fig 12: AE2ED experienced between each of the three sensors and node 5

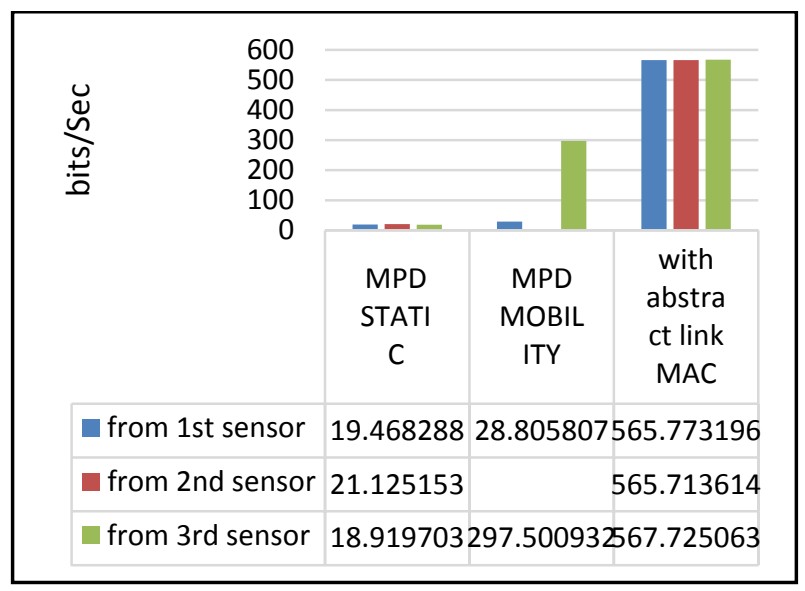

Fig 13: Unicast received throughput from each sensor

Additionally, the total IPv6 packets generated and Routing Overhead (RO) messages received have approximately doubled in MPDs mobile scenario against MPDs static scenario, where they were 407 Packets and 96 messages in the latter scenario, then they rose to 701 packets and 370 messages in the former.

In contrast, both of these parameters (IPv6 packets generated and RO messages received) had been decreased in the third scenario via the provision of WALM, as what can be seen in Figures 14 and 15 respectively, even lower than the lowest values of the first scenario when MPDs were static.

Despite the increment in the last two parameters due to MPDs mobility, and different from what is already known in MANET and was confirmed via the results obtained in the first section of this chapter, the delivery ratio has been improved by the mobility of the MPDs nodes in this design, which presents an opportunistic collaborative that should not be neglected and that can be used in IoT.

The other obtained advantage was through the decrement in AE2ED via mobility, which is another interesting and unusual in MANET, and that might be as a result of transmitting through only one hop in the MPDs mobility scenario, instead of multi-hops in the MPDs static scenario. Where, in the first scenario, the only delay experienced will be a delay of one hop and one node queuing when MPDs receives the data messages from the sensors when they move near them and deliver these messages to the sink node. Whilst, in the second 
scenario, the data messages will suffer from being transmitted over multi-hops as well as each hop's queuing time. However, in the MPDs mobile scenario, the mobility speed and the time spent by each of the MPDs nodes to reach the sink node's (node 5) coverage area will be crucial and will play a critical role. Therefore, it must be taken into consideration.

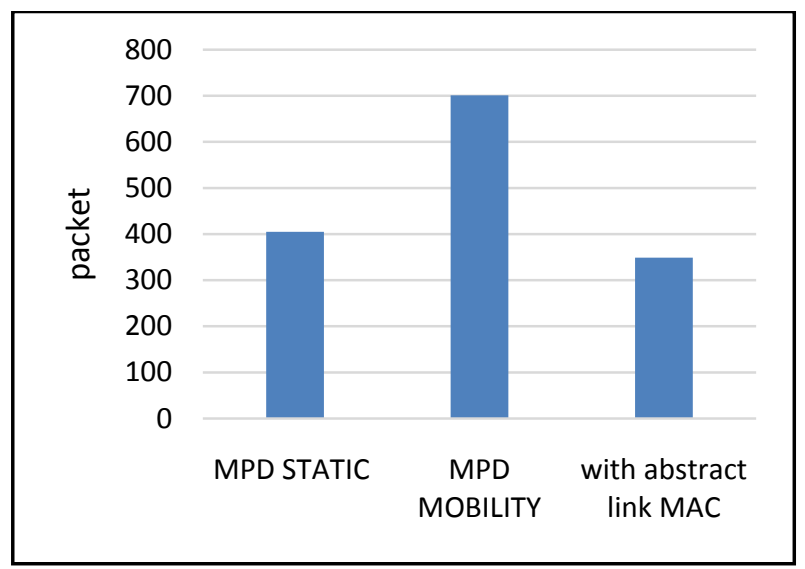

Fig 14: Total IPv6 packet generated for all nodes

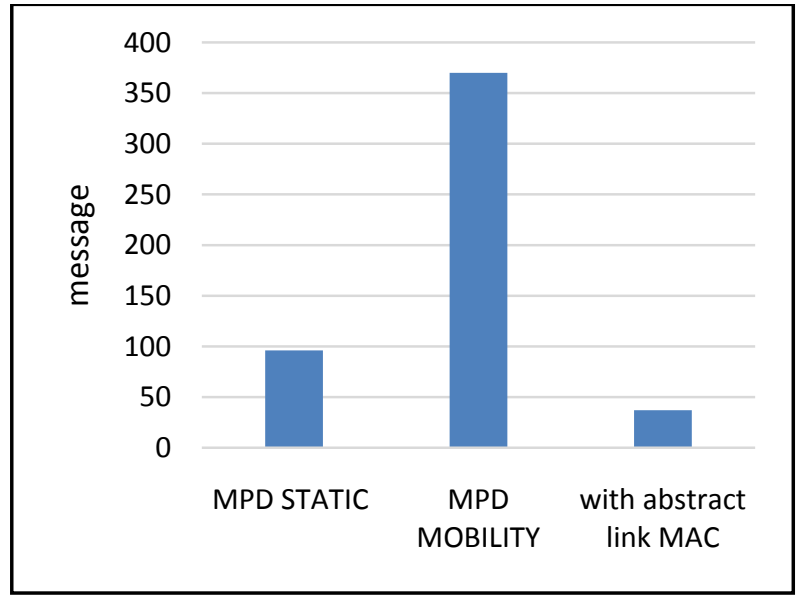

Fig 15: Total RO messages received for all nodes

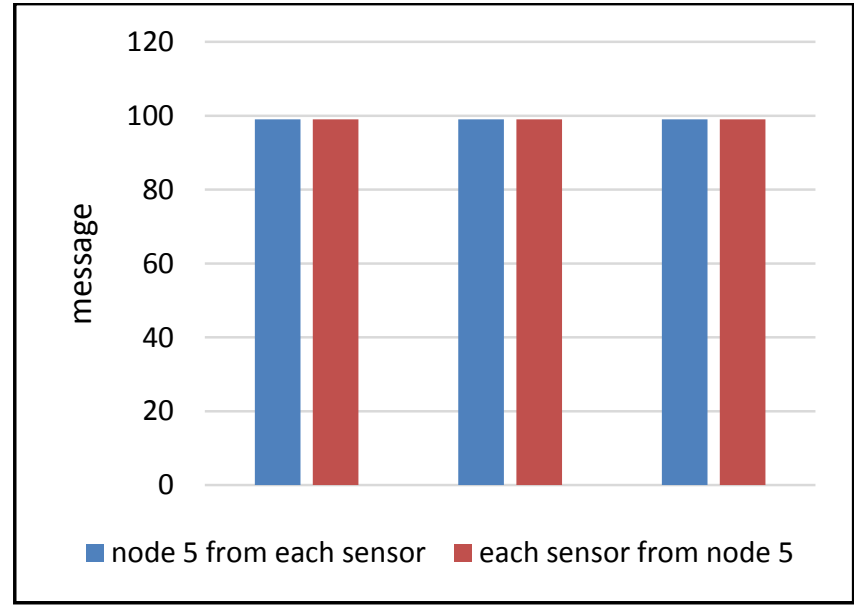

Fig 16: Total Unicast Messages Received in Both Sides

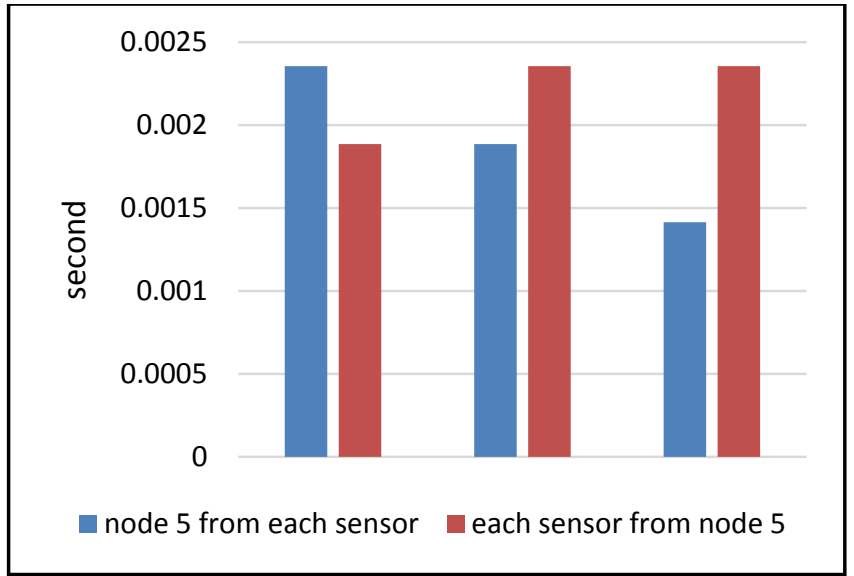

Fig 17: AE2ED for sink node and the three sensors

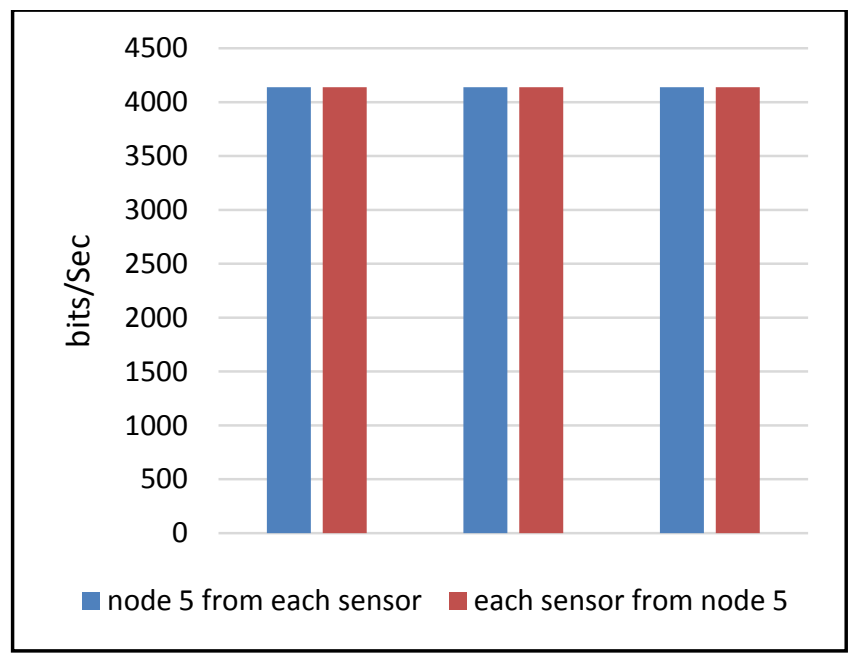

Fig 18: Unicast received throughput for node 5 and the three sensors

In the last scenario, when CBRs have been deployed to transmit from the sink node to the sensors, besides the original CBRs, which transmit in the other direction (from sensors to node 5), it is noticeably seen that communication offered high reliability in the number of data messages received without a need to involve the MPDs in the communication, as in Figures 16, 17, and 18, and Table 2 respectively.

It is even possible to disable fragmentation in IPv6 configuration, and the connections will be maintained, but the packets will be dropped from the MPDs as soon as they are involved in the communications. Hence, to maintain the redundancy of the network in case any of the assumed devices has gone out of service or has left the communications coverage area. Fragmentation kept enabled, to allow MPDs working, where the dependency will be over MPDs again.

TABLE 1 Other parameters for last IH scenario

\begin{tabular}{|l|l|}
\hline $\begin{array}{l}\text { Total IPv6 packet generated by all } \\
\text { nodes }\end{array}$ & 691 packets \\
\hline $\begin{array}{l}\text { Sum of time spent transmitting } \\
\text { for all nodes }\end{array}$ & 0.018788 second \\
\hline $\begin{array}{l}\text { Total RO messages received for } \\
\text { all nodes }\end{array}$ & 82 messages \\
\hline
\end{tabular}


The greatest advantage gained from the $\mathrm{IH}$ design is the remarkable extension that has been achieved in the sensors' communication area against the normal ZigBee or any other pure WSNs. Besides through providing WALM the ability to transmit from the nodes that work on IEEE 802.15.4 standard to those that work over IEEE 802.11 directly, without needing MPDs to be configured. While, in terms of the proposed WALM absence, the higher the MPDs density, the better the data messages delivery ratio will be, and vice versa.

These pros of the IH design do not prevent or obscure some of the cons. One example is the inability of IEEE 802.15.4 to handle headers longer than 127 bytes, as well as the IPv6 mechanism via its MTU, which will be transmitted even if it has not been fully used, if the fragmentation unit has not been reduced.

Last but not least, this kind of novelty that is presented by the ability of mobile nodes to provide WALM when they come between two of the discussed different IEEE 802 standards coverage area can provide advantages for both standard to benefit from each other.

\section{CONCLUSION}

MANET tends to be the essential technology in IoT. Furthermore, applying IPv6 seems to be inevitable due to running out of available IPv4 addresses. The converging between the two different IEEE 802 standards, which are both considered to be a part of Ad-hoc networks, by using IPv6 is not at first as simple as plug and play because of the differences between PHY and MAC layers between both standards. The second reason is due to the criteria of IPv6 and its MTU values. Where, because of WSN, which works on IEEE 802.15.4 standard and has a limited header size with 127 bytes as maximum, as well as the 1280 bytes in the MTU in IPv6. A special configuration was needed, and the fragmentation threshold required changing, as what had been configured.

Additionally, MANET, which works on IEEE 802.11, cannot transmit and receive directly from any WSN nodes. Therefore, MPD nodes have been configured to relay both standards' traffic when they became between the two standards' coverage areas.

This integration succeeded in creating a collaboration between these different standards. That collaboration can extend the communication area and that will, in turn, lead to extending the area monitored by sensors. In addition, it might work out of the role that mobility negatively influences on performance, as it has been seen that when MPDs were mobile, the delivery ratio was higher than when they were static. Even if that cannot be a crystal role but it can be taken into consideration when the design and implementation of the network take place.

The reason behind using MPDs and configuring the scenario platform to work with double channels as well as the special configuration of IPv6 fragmentation threshold and double IP configuration in the cloud that connects sensors is to allow such integration because of the non-availability of using 6LoWPAN, which is a standard presented by IETF to enable IEEE 802.15.4 standard to work over IPv6 in QualNet simulator. Besides, the unavailability of supporting IPv6 in the aforementioned cloud forced us to tunnel IPv6 over IPv4 in that precise side.

Last but not least, when the same assumption of the device that provided a WALM was deployed, the delivery ratio and throughput soared up again in comparison with the design when these devices were not used. Furthermore, it allowed direct communication between the different standards in both directions without the need of MPDs to be involved.

All what have been mentioned lead us to the opportunistic advantages that can be gained due to the integration of different standards to work together, which can even reduce the poorness in the Ad-hoc routing protocols' performance in many areas. However, these routing protocols, in their turn, need to be modified and enhanced as much as possible, mainly to reduce their overhead signaling, which would lead to the ability of obtaining the suprior possible performance, which strongly needed in IoT, where the reliability of the data is critical and decisive.

In addition, IPv6 is not the absolute solution for all IPv4 problems. Despite the enhancement in the routing techniques via ND, it may overload the network with its long headers. Likewise, it brings the need for new standards to be innovated in order to allow some IEEE standard to work with it.

\section{FUTURE WORK}

Hence, future work tends to focus on converging MANET, WSN, and LTE, as a trying to examine the ability to extend the heterogeneity of more different standards by trying to connect the sink node that works on IEEE 802.11 to an LTE device or an eNodeB directly. Alternatively, future work can involve how to extend the coverage area of MANET nodes by involving LTE technology in the communication to allow everything connected to the internet to communicate with each other, and to take the maximum possible advantage of each other.

\section{REFERENCES}

[1] Reina, Daniel G., Sergio L. Toral, Federico Barrero, Nik Bessis, and Eleana Asimakopoulou. "The Role of Ad Hoc Networks in the Internet of Things: A Case Scenario for Smart Environments." In Internet of Things and Inter-Cooperative Computational Technologies for Collective Intelligence, pp. 89-113. Springer Berlin Heidelberg, 2013.

[2] Jara, Antonio J., Latif Ladid, and Antonio F. GómezSkarmeta. "The Internet of Everything through IPv6: An Analysis of Challenges, Solutions and Opportunities." JoWUA 4, no. 3 (2013): 97-118.

[3] Grajzer, Monika, and Mariusz Glabowski. "On IPv6 experimentation in wireless mobile ad hoc networks." Journal of Telecommunications and Information Technology 3 (2014): 71.

[4] Racherla, S., and Daniel, J., 2012. IPv6 Introduction and Configuration, (pp. 10-29) IBM.

[5] Institute of Electrical and Electronics Engineers. IEEE Std 802.15.4-2006, Wireless Medium Access Control (MAC) and Physical Layer (PHY) Specifi- cations for Low-Rate Wireless Personal Area Networks (WPANs), 8 September 2006.

[6] Institute of Electrical and Electronics Engineers. IEEE Std 802.15.4- 2006, Wireless Medium Access Control (MAC) and Physical Layer (PHY) Specifications for Low-Rate Wireless Personal Area Networks (WPANs), 8 September 2006.

[7] Li, Weibo, Harsha Sirisena, and Krys Pawlikowski. "An address-based routing scheme for static applications of wireless sensor networks." InTelecommunication 
Networks and Applications Conference, 2007. ATNAC 2007. Australasian, pp. 371-376. IEEE, 2007.

[8] Masood, Syed Haani. "Performance comparison of IEEE $802.11 \mathrm{~g}$ and IEEE $802.11 \mathrm{n}$ in the presence of interference from 802.15. 4 networks." ArXiv preprint arXiv:1308.0678 (2013).

[9] Bellavista, Paolo, Giuseppe Cardone, Antonio Corradi, and Luca Foschini. "Convergence of MANET and WSN in IoT urban scenarios." IEEE Sensors Journal 13, no. 10 (2013): 3558-3567.

[10] Bai, Yong, Wencai Du, Zhengxin Ma, Chong Shen, Youling Zhou, and Baodan Chen. "Emergency communication system by heterogeneous wireless networking." In Wireless Communications, Networking and Information Security (WCNIS), 2010 IEEE International Conference on, pp. 488-492. IEEE, 2010.

[11] Cardone, Giuseppe, Antonio Corradi, and Luca Foschini. "Reliable communication for mobile MANET-WSN scenarios." In Computers and Communications (ISCC), 2011 IEEE Symposium on, pp. 1085-1091. IEEE, 2011.

[12] Radmand, Pedram, Alex Talevski, Stig Petersen, and Simon Carlsen. "Comparison of industrial WSN standards." In 2010 4th IEEE International Conference on Digital Ecosystems and Technologies (DEST), IEEE, Dubai, United Arab Emirates, pp. 632-637. 2010.

\section{AUTHOR'S PROFILE}

Mahmood I. Alsaydia BSc. In computer engineering (2005) from university of Mosul / Iraq. Then work as IT and computer engineer In Nenivah governorate (2006). After that, I was a senior engineer and the manager of the computer department in Nineveh pensioner office (2007-2014)

MSc. In Data Telecommunications \& Networks (2016) from University of Salford / UK.

Currently working for Danish refugee Council. 